\title{
IoT based Smart Garbage System Powered with Solar Cell
}

\author{
Ms. C. Vinothini ${ }^{[1]}$ \\ Assistant Professor, \\ Department of Computer Science and Engineering, \\ Dr. N. G. P. Institute of Technology, Coimbatore, India. \\ Tharini $\mathrm{M}^{[2]}$, Saranya Sasikumar $\mathrm{G}^{[3]}$, Ikash $\mathrm{K}^{[4]}$, Karthick $\mathrm{R}^{[5]}$ \\ Final year Students, \\ Department of Computer Science and Engineering, \\ Dr. N. G. P. Institute of Technology, Coimbatore, India.
}

\begin{abstract}
This paper presents IoT based project of a smart garbage bin which has a real time monitoring system that integrates multiple technologies which includes the sensors, solar panel and arduino. The main aim of this project is to offer a cost-effective and efficient waste collection and management system in order to provide a well maintained and healthy environment. This system provides a mechanism for remote monitoring of solid waste bin in the real-time enabled with the Wi-Fi connection, to assist the waste management activity. The proposed system is dependent on wireless sensor network [WSN]. In this System there are three modules: Hardware Interface, Communication Interface, Data storage and verification. The proposed IOT based smart, real-time garbage monitoring system makes use of a wide range of technologies which will yield a green and healthy lifestyle which results in a better living environment for mankind.
\end{abstract}

Keywords: Wi-Fi connection, solar cell, rain sensor, ultrasonic sensor, cost-effective, arduino, garbage monitoring, efficient, Internet of things and waste management.

\section{INTRODUCTION}

In our country disposing the garbage is a serious issue. Even educated people tend to throw the garbage outside the garbage tanks, this is due the presence of insufficient garbage tanks near the road sides. In the cities most of the road ends having garbage tanks, and most of the people use the garbage tanks in the rightful manner. But now the problem is when the garbage tanks over flow it smells a lot during rainy season. This issue leads to very speedy spread of diseases to the nearby places. In order to avoid this problem, the waste garbage will be sent for burning. But burning the garbage in incinerators releases highly dangerous gases, ash and dust which contributes to global warming and it pollutes natural bodies.

In India, the average resident produces seven and a half pounds of garbage every day. In recent times people face critical trouble in saving the weakening environment, especially in rural areas, where solid wastes are dumped which overflows on streets. It is observed that cities have no proper controlled structure for garbage disposal. Each year, millions of dollars are spent on picking up the litter. As new technology developed and with modern equipments, the level of waste produced increases every day. The proportion of chemicals in the waste is increasing with the hectic pace of the modern development. With the use of plastic, a problem has arisen, the plastic never degrades. In order to overcome this issue, we are proposing the "IoT based smart garbage system powered with solar cell" which is developed for both rural and urban areas to solve the garbage disposal related problems.

\section{LITERATURE REVIEW}

There are multiple solutions proposed for the waste management provided with IoT facilities that have been proposed and invented in the literature which helps the solid waste management authorities improve the quality of service delivery.

Researchers in [6] made use of the level Sensor to monitoring the level of waste-bin load sensor to measure weight of the bin. In the [7] Ultra sonic sensors are used to detect level of waste and force sensors are used to detect weight. Dr. Mrs. Neeta Anilkumar Doshi in [8] implemented a system in which it keeps dry and wet garbage separately so that different processes- composting, recycling, incineration shall be applied to different kinds of garbage.

From [9] Himadri Nath Saha included Ultra sonic sensors which are used to detect the level of waste. Also force sensors are used to detect weight of the garbage dumped in the tanks. Sahil Mirchandani in [10] used level sensor and toxicity sensor which will be sending the data collected at intervals of 15 minutes each to the monitoring station. In [11] If the user is authentic it grants the permission to the micro controller to open the bin which uses cloud based implementation.

\section{METHODOLOGY}

Several problems in waste management will be considered in order to propose the new framework.

1) A mechanism to close the lid of dustbin in case of rain is implemented in the proposed system. This avoids bad smell while nearing the garbage. Also the waste remains dry.

2) The required number of additional dustbins to adjust the load is introduced. 
3) The filling rate of each of the dustbins is maintained in cloud.

4) Periodic notifications to the monitoring stations about the status of the garbage in the tanks.

5) Battery is powered with the solar cell for efficient energy usage.

6) This tank is fully sensor integrated.

7) Ultra Sound have been used for tank level indicators. There are 3 steps of indication is available to view the level of tank. $25 \%, 50 \%$ and $75 \%$, this information will be sent the municipality / Corporation for speedy tank disposal.

8) In case of installing smart dustbin in rural areas, the dustbin will be integrated with a solar panel with LED light for dustbin visibility during night times (optional)

The proposed system provides the above stated functionalities by making use of the IoT devices and the cloud for storage of data. Figure 1 shows the proposed system framework. This framework consists of three modules namely: Hardware Interface, Communication Interface, Data storage and verification.

Once the garbage tanks start to fill the rate of filling is calculated and based on that estimated number of garbage tanks can be employed in that region. In case of rain, the tank will be closed automatically. The order in which the tanks must be cleaned is also intimated to the monitoring station. The solar cells are used for battery and with the help of arduino and Wi-Fi connection the garbage tank can be monitored periodically and the information is stored in cloud.

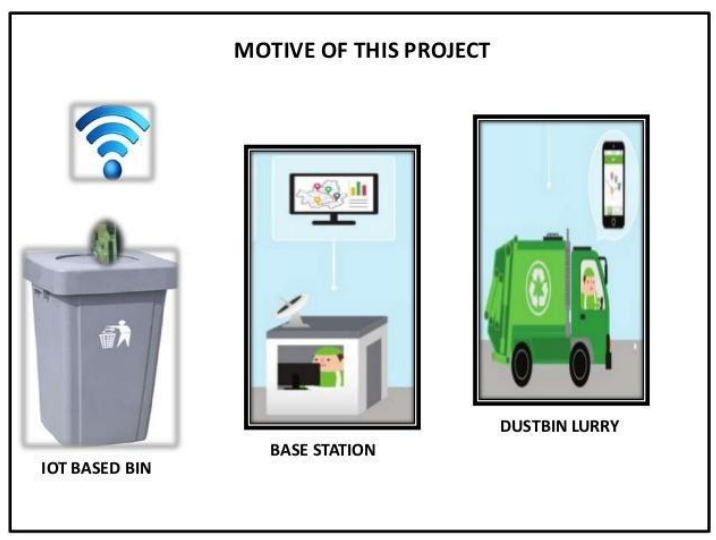

Fig. 1

III.I. HARDWARE INTERFACE

Hardware interface is the initial Module in the project. This module comprises of sensors and other hardware components. The PIC or Arudino Microcontroller is used to provide efficient sensor interface. There are multiple sensor interface that are implemented in this model. All the sensors are being embedded with the LCD for the purpose of offline communication. Microcontroller is connected with the power supply unit which is a solar powered battery.

The hardware interface includes the following components: Arduino uno, Solar panel, Ultrasonic sensor, Raindrop sensor, Battery, Node MCU and the Mechanical setup.

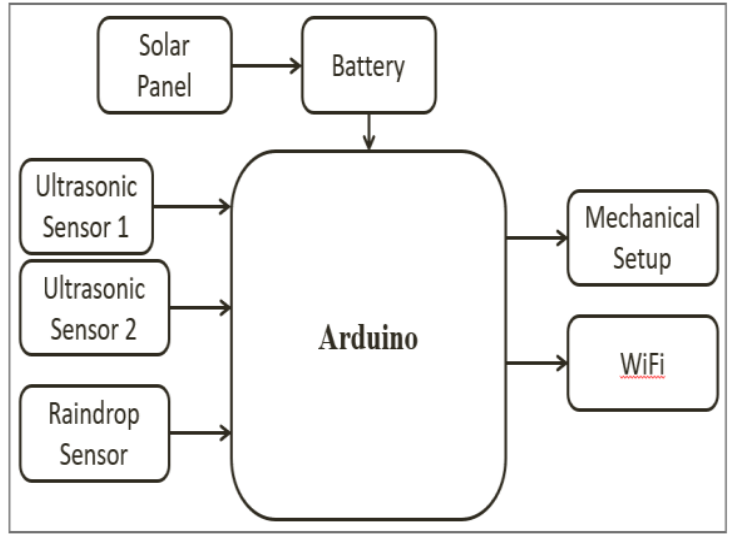

Fig. 2

\section{III.I. I. INTERFACING RAIN SENSOR}

The rain sensor is a tool used for rain detection purpose. It acts as a switch when raindrop falls through the raining board and it is also used for measuring intensity of the rainfall.

It consists of a rain board and a control board, power indicator LED and an adjustable sensitivity feature though a potentiometer.

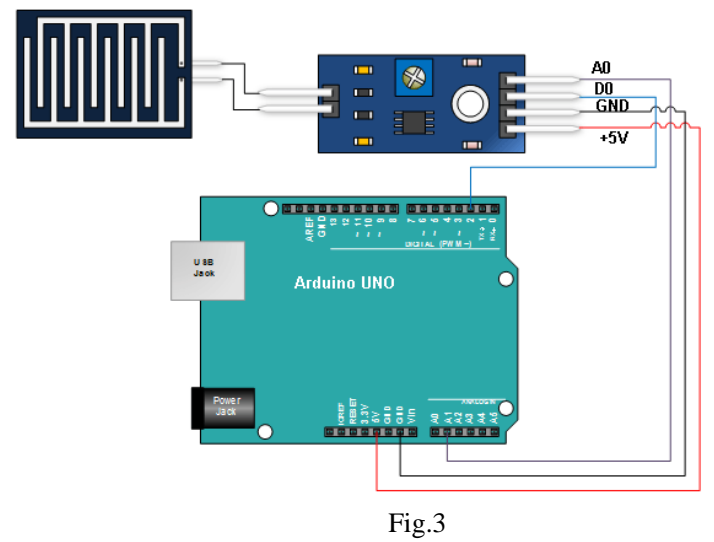

Rain sensor adopts to high quality of RF-04 double sided material whose area is given by $5 \mathrm{~cm} \times 4 \mathrm{~cm}$ nickel plate on sides. It has features such as Anti-oxidation, anticonductivity, with long use time.

Comprising of Comparator output signal with clean waveform, driving ability, over $15 \mathrm{~mA}$. Potentiometer is used to adjust the sensitivity. The sensor works with a voltage of $5 \mathrm{~V}$ with output format in Digital switching output (0 and 1) and analog voltage output AO.

\section{III.I.II. INTERFACING ULTRASONIC SENSOR}

There is provided an ultrasonic diagnostic system where the ultrasonic probe is detachably connected thereto, and ultrasonic waves gets transmitted from ultrasonic probe into the subject in order to obtain received signals from ultrasonic waves reflected within the subject, thereby displaying diagnosis of image carrying information based on the received signals. It is also provided that ultrasonic module including a processing circuit for the received signals, being used in the ultrasonic diagnostic system. 
The ultrasonic diagnostic system has a higher level of computer support. The ultrasonic module is connected through the general-purpose which is interfaced to a computer system. Ultrasonic module has a main element, an analog unit for performing an analog signal processing and it is connected to another computer system.

\section{III.I.III. ARDUINO SETUP}

The major tool used in the IoT based smart garbage system is the Arduino. It is used to write and upload the programs to Arduino compatible boards. Arduino IDE is a crossplatform application which is written in functions from $\mathrm{C}$ and $\mathrm{C}++$ languages.

There are two processes performed one is fork of Arduino IDE and the other one is IDE with Board Manager.

The figure 4 shows how the arduino is connected along with the two ultrasonic sensors and the Wi-Fi module with the pin connections. Once the process to be performed is coded, it is uploaded to the arduino device and the sensed data from sensors are collected and transferred through the Wi-Fi channel.

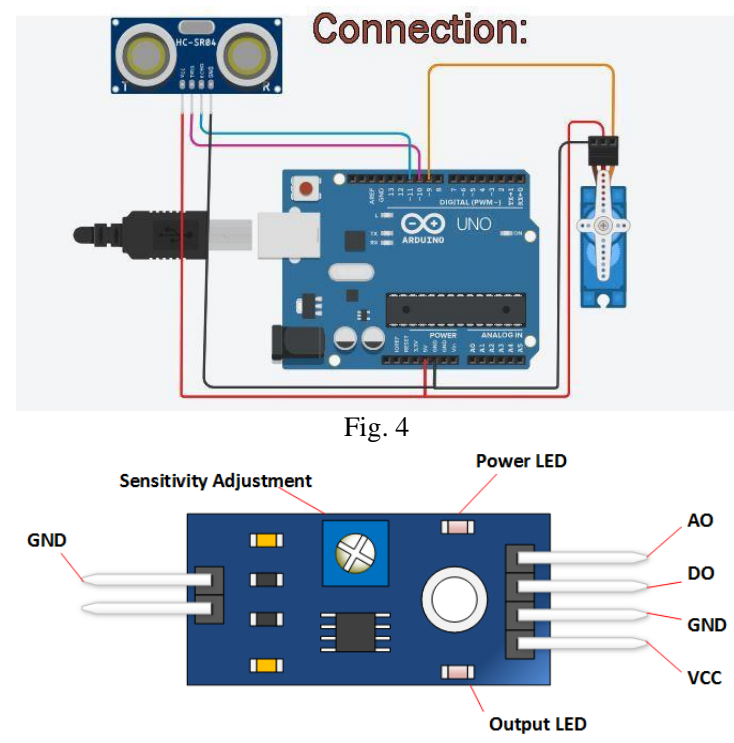

Fig. 5

\section{III.I.IV. SOLAR PANEL}

Solar panel is implemented to power the batteries in the proposed system. These Solar panels acts as a way to convert solar power into electrical power. It converts the energy instantly or heats the water with the amount of produced energy. Photo-voltaic cells are the ones made by using the semiconductor structures where the sun beam gets absorbed where electrons are emitted from the atoms to which it is bounded.

By the release process, a current is generated. Photovoltaic is called as the process happening between the beam absorbed by the cell and the electricity induced. By implementing a common principle, the solar power is converted into the electric power.

Solar batteries are the ones produced by waffling of the $p-n$ semi-conductors. Under the beam, electron flows and the current is generated. In case of closed-loop, the Photovoltaic current gets passed through the external load. Where as in case of open-loop, the current follows into the circuit through the $p-n$ diode structure. Solar batteries are represented with the equivalent circuit from a current source, a resistor and a diode in parallel with an external load resistor.

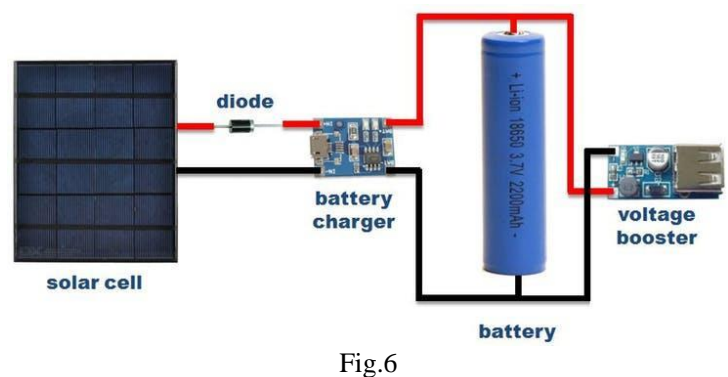

\section{III.II. COMMUNICATION INTERFACE}

COMM port is usually the communication port. This module interfaces the hardware unit with PC. The communication has been done with the help of RS232 tool. RS232 is a system interface tool that efficiently works by converting the machine language into the system language. Using Hyper terminal, data from sensor unit is sent to PC where the data is split sensor wise before transferring into PC.

In order to implement IOT, the data collected from sensor is mainly used. A set of collected data from the corresponding sensors is used as a data set. Uploading is the transmission of a file from one computer system to another which is usually larger computer system. From the network user's point-of-view, to upload a file is to send it to another computer which is set up to receive the information. Uploaded data will then be stored in the web server. In the proposed system we upload the data set in a cloud server.

\section{III.III. DATA STORAGE AND VERIFICATION}

The public cloud environment is usually the IaaS/PaaS Infrastructure or Platform as a Service which we rent from the Linux (IaaS) or Microsoft (PaaS). Both are enabled for the purpose of web hosting. SaaS stack runs under the Internet environment most likely in a virtualized one which would make it look private. In the proposed system we make use of private cloud technology.

Here we execute in a cloud environment. We implement web services for the output purpose. Thus SaaS can be fully utilized in the cloud environment as IaaS/PaaS.

The data verification is a user defined function model, it is said to be the application layer, where all the data were verified and viewed by the users and admin.

Based on the architecture design, user and admin will be available in the application layer where as business layer is the decision making layer and all data will be available in the data layer.

\section{SUMMARY}

This paper presents a model of IoT innovation project for the purpose of waste management system. This proposed system provides efficiency of waste bin collection activities and cost reduction and helps from the overflow issues in the garbage tank, Additional 
number of garbage tanks to be incorporated in the particular region can be known and Proper notifications to the monitoring authority helps in effective maintenance and clearance of waste from the garbage tanks which ensures a healthy lifestyle to people.

\section{REFERENCES}

[1] Dr.N.Sathish Kumar, B.Vijayalakshmi, R. Jeniferprarthana, A .Shankar. IOT Based Smart Garbage alert system using Arduino UNO, 2016 IEEE.

[2] Sagnik Kanta, Srinjoy Jash, Himadri Nath Saha. Internet Of Things Based Garbage Monitoring System, 2017 IEEE.

[3] Himadri Nath Saha, Samabrita kundu, Sourav Gon, Sumandrita Moitra, Annesha Nayak. Iot Based Garbage Monitornng and Clearance Alert System. 2018 IEEE.

[4] Sourangsu Mukherjee, Budhaditya Bhattacharyya and Neelkantha Banerjee. Harnessing Green Energy for Smart Dustbin, 2017 IEEE International Conference on Smart Technologies and Management for Computing, Communication, Controls, Energy and Materials (ICSTM).

[5] Murugaanandam. S, Ganapathy. V and Balaji. R. Efficient IOT Based Smart Bin for Clean Environment. International Conference on Communication and Signal Processing, April 3-5, 2018, India

[6] Aksan Surya Wijaya, Zahir Zainuddin, Muhammad Niswar Design a Smart Waste Bin for Smart Waste Management, IEEE 5th International Conference on Instrumentation, Control, and Automation (ICA), 2017

[7] S. Vinoth Kumar, T. Senthil Kumaran, A. Krishna Kumar and Mahantesh Mathapati - Smart Garbage Monitoring and Clearance System using Internet of Things, IEEE International Conference on Smart Technologies and Management for Computing, Communication, Controls, Energy and Materials (ICSTM), 2017.

[8] Sudharani Ashok Ghadage, Dr. Mrs. Neeta Anilkumar Doshi - IoT Based Garbage Management (Monitor and Acknowledgment) System: A Review, Proceedings of the International Conference on Intelligent Sustainable Systems (ICISS 2017) IEEE Xplore Compliant, 2017.

[9] Himadri Nath Saha, Sourav Gon, Annesha Nayak - Iot Based Garbage Monitoring and Clearance Alert System, (ICISS 2018) IEEE Xplore Compliant, 2018.

[10] Sahil Mirchandani, Sagar Wadhwa - IoT Enabled Dustbins, IEEE, International Conference on Big Data, IoT and Data Science (BID), 2017.

[11] Aayush Tripathi, Chinmay Pandey, Ankur Narwal - Cloud Based Smart Dustbin System for Metro Station, IEEE Xplore Compliant (ICISS 2018). 